\title{
Treatment with Delipid Extracorporeal Lipoprotein Filter from Plasma after Intravenous Thrombolysis for Acute Ischemic Stroke: A Single-Center Experience
}

\author{
Yu Cuia, b Zhong-He Zhou ${ }^{a}$ Xiao-Wen Hou ${ }^{a}$ Hui-Sheng Chen ${ }^{a}$ \\ a Department of Neurology, General Hospital of Northern Theater Command, \\ Shenyang, China; ${ }^{\mathrm{b}}$ Department of Life Science and Biopharmaceutics, Shenyang \\ Pharmaceutical University, Shenyang, China
}

\author{
Keywords \\ Delipid extracorporeal lipoprotein filter from plasma Acute ischemic stroke $\cdot$ Intravenous \\ thrombolysis - Mechanism
}

\begin{abstract}
Introduction: The delipid extracorporeal lipoprotein filter from plasma (DELP) has been approved for the treatment of acute ischemic stroke (AIS) by the China Food and Drug Administration, but its effectiveness and mechanism are not yet fully determined. The purpose of this study was to evaluate the effect of DELP treatment on AIS patients after intravenous thrombolysis. Methods: A retrospective study was performed on AIS patients with no improvement within $24 \mathrm{~h}$ after intravenous thrombolysis who were subsequently treated with or without DELP. Primary outcome was the proportion with a modified Rankin scale (mRS) of 0-1 at 90 days. Secondary outcomes were changes in National Institute of Health Stroke Scale (NIHSS) score from $24 \mathrm{~h}$ to 14 days after thrombolysis, and the rate of improvement in strokeassociated pneumonia (SAP). The main safety outcomes were the rates of symptomatic intracranial hemorrhage and mortality. To investigate its mechanisms, serum biomarkers were measured before and after DELP. Results: A total of 252 patients were recruited, 63 in the DELP group and 189 matched patients in the NO DELP group. Compared with the NO DELP group, the DELP group showed an increase in the proportion of $\mathrm{mRS} 0-1$ at 90 days $(p=0.042)$. More decrease in NIHSS from $24 \mathrm{~h}$ to 14 days $(p=0.024)$, a higher rate of improvement in SAP ( $p=$ $0.022)$, and lower mortality $(p=0.040)$ were shown in DELP group. Furthermore, DELP decreased levels of interleukin (IL)-1 $\beta$, E-selectin, malondialdehyde, matrix metalloprotein 9, to-
\end{abstract}


tal cholesterol, low-density lipoprotein, and fibrinogen, and increased superoxide dismutase $(p<0.05)$. Conclusions: DELP following intravenous thrombolysis should be safe, and is associated with neurological function improvement, possibly through multiple neuroprotective mechanisms. Prospective trials are needed.

\section{Introduction}

Intravenous thrombolysis (IVT) with recombinant tissue plasminogen activator (rtPA) has been demonstrated as a standard treatment and is strongly recommended for the early management of patients with acute ischemic stroke (AIS) [1]. However, IVT is not satisfactory in clinical practice; only $30 \%$ of patients can acquire good prognosis, and 1-3 \% patients experience symptomatic intracranial hemorrhage and even mortality in moderate-to-severe cases $[2,3]$. It has been a hot topic how to improve the prognosis of thrombolytic patients with AIS. Early neurological improvement (ENI) was defined as an NIHSS score of 0 or 1 at 24 $\mathrm{h}$, or an improvement of $\geq 8$ points compared to baseline [4]. Previous studies suggested that ENI was important and closely related to favorable outcome at 3 months $[4,5]$.

Heparin-induced extracorporeal low-density lipoprotein (LDL) precipitation (HELP) is a nonpharmacological therapy for hyperlipidemia and atherosclerotic disease, acting through multiple mechanisms such as reducing total cholesterol (TC), triglyceride (TG), LDL, fibrinogen (FIB), C-reactive protein (CRP), vascular cell adhesion molecule (VCAM), ICAM, prothrombin, Factors V-XIII, D-dimer, and prothrombin fragment [6, 7]. With a similar mechanism to HELP, the delipid extracorporeal lipoprotein filter from plasma (DELP) has been found to improve neurological function and the ability to perform activities of daily life in AIS patients, and it has been approved for the treatment of AIS by the China Food and Drug Administration (CFDA) [8].

To date, no study has investigated the efficacy and safety of DELP treatment for AIS patients after IVT. Given the possible multiple mechanisms of DELP, we argue that DELP treatment may improve the outcome of AIS patients who did not improve within 24 after IVT. This study was designed to investigate the efficacy and safety of DELP treatment in this population. Furthermore, a series of serum biomarkers were measured to determine the mechanisms of DELP.

\section{Materials and Methods}

\section{Study Design and Patient Selection}

From a prospective database, we continuously collected the patients with AIS receiving IVT at General Hospital of Northern Theater Command from January 2012 to December 2016 according to the inclusion and exclusion criteria. Patients were included if they met the following criteria: (1) AIS, (2) on IVT with rtPA $(0.9 \mathrm{mg} / \mathrm{kg}$, maximum $90 \mathrm{mg}$; Boehringer Ingelheim Pharma GmbH \& Co), and (3) no decrease in National Institute of Health Stroke Scale (NIHSS) score $24 \mathrm{~h}$ after receiving IVT. Patients were excluded if there was: (1) $>4.5 \mathrm{~h}$ between the onset of neurological symptoms and IVT treatment, (2) endovascular intervention, (3) a lack of clinical data.

According to the treatment regimen, patients were divided into 2 groups. The DELP group received DELP treatment within 1 week following IVT and the NO DELP group did not receive DELP treatment. Propensity score matching (PSM) was performed between groups with the ratio 1:3, and a nearest-neighbor matching strategy was applied with $R$ software

\section{Karger'}


v3.6.3. PSM was operated with control factors including age, gender, systolic blood pressure (SBP), diastolic blood pressure (DBP), symptom onset-to-thrombolysis time (OTT), NIHSS score at admission and $24 \mathrm{~h}$ after IVT, the Trial of Org 10172 in Acute Stroke Treatment (TOAST) classification, and the patient's medical history.

\section{DELP Treatment Procedure}

As described in a previous study, the venous channel was established by selecting the bilateral medial forearm [8]. The blood was pumped into the PCS2 plasma separator through one side of the forearm vein. The plasma was separated by pump and then sent to the DELP system (Shanghai Jiangxia Blood Technology Co.). After purification, the plasma was returned to the patient via another forearm vein. The total treatment plasma volume was $800-1,000 \mathrm{~mL}$; the anticoagulant $4 \%$ sodium citrate dehydrate solution with a ratio of $1: 16$ to plasma, was dropped before pumping. To prevent hypocalcemia, $500 \mathrm{mg} \mathrm{CaCl} 2$ diluted with $250 \mathrm{~mL}$ physiological saline was infused at a rate of $150 \mathrm{~mL} / \mathrm{h}$, in the first cycle of the returning blood transfusion.

\section{Laboratory Determinations}

To investigate the mechanisms of DELP, the levels of interleukin (IL)-1 $\beta$, IL-6, tumor necrosis factor (TNF) $\alpha$, VCAM1, E-selectin, IL-18, matrix metalloprotein 9 (MMP-9), plasminogen activator inhibitor-1 (PAI-1), soluble CD40 ligand (sCD40L), malondialdehyde (MDA), and superoxide dismutase (SOD) in blood samples were measured in 8 patients. We also measured the change in levels of total cholesterol (TC), triglyceride (TG), high-density lipoprotein (HDL), LDL, and FIB before and after DELP treatment in 40 patients.

Approximately $4 \mathrm{~mL}$ of venous blood were obtained for measurement $1 \mathrm{~h}$ before and $2 \mathrm{~h}$ after DELP treatment, respectively. The blood samples were centrifuged at $1000 \mathrm{~g}$ for $10 \mathrm{~min}$ at $4{ }^{\circ} \mathrm{C}$. The serum blood samples were transferred into a $1.8-\mathrm{mL}$ cryotube and stored at $-80^{\circ} \mathrm{C}$. The determinations of IL-1 $\beta$, IL-6, TNF $\alpha$, VCAM1, E-selectin, IL-18, MMP-9, PAI-1, sCD40L, MDA, and SOD were performed by ELISA using the Quantikine kit (R\&D Systems, Abingdon, UK). The determinations of TC, TG, HDL, LDL, and FIB were performed by automatic analyzer (Hitachi 7170, Tokyo, Japan).

\section{Data Collection}

The following data were obtained from an electronic database: age, gender, smoking status, alcohol consumption, history of hypertension, diabetes mellitus, coronary artery heart disease, stroke, atrial fibrillation, BP, NIHSS score (at admission, $24 \mathrm{~h}$ after IVT, and 14 days after hospitalization), OTT, TOAST classification, gastrointestinal bleeding, symptomatic intracranial hemorrhage ( $\mathrm{sICH}$ ), mortality, and modified Rankin scale (mRS) 90 days after onset.

For patients with stroke-associated pneumonia (SAP), the counts of white blood cells, lymphocytes, and neutrophils, and the CRP concentration in the peripheral blood in the 2 groups were also recorded.

\section{Outcome Assessment}

The primary efficacy outcome was a favorable functional outcome at 90 days (a score of $0-1$ on the $\mathrm{mRS}$ ). Secondary efficacy outcomes were distribution in mRS and a reduced NIHSS score in the period from $24 \mathrm{~h}$ to 14 days after thrombolysis. The primary safety outcome was death due to any cause during hospitalization. Other safety outcomes included sICH and gastrointestinal bleeding during hospitalization. According to the European Cooperative Acute Stroke Study (ECASS) III definition, sICH was defined as any intracranial hemorrhage associated with clinical deterioration (an increase of $\geq 4$ points on the NIHSS or death) [1]. Gastrointestinal bleeding was defined as any evidence of gastrointestinal bleeding.

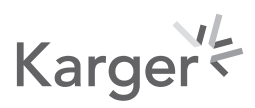




\section{Cerebrovascular Diseases Extra}

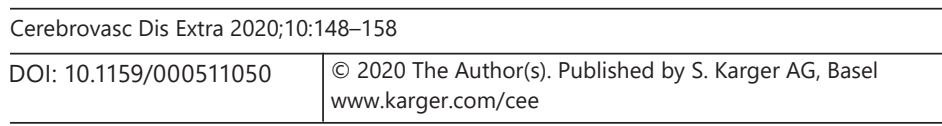

Cui et al.: DELP Treatment after Intravenous Thrombolysis for AIS

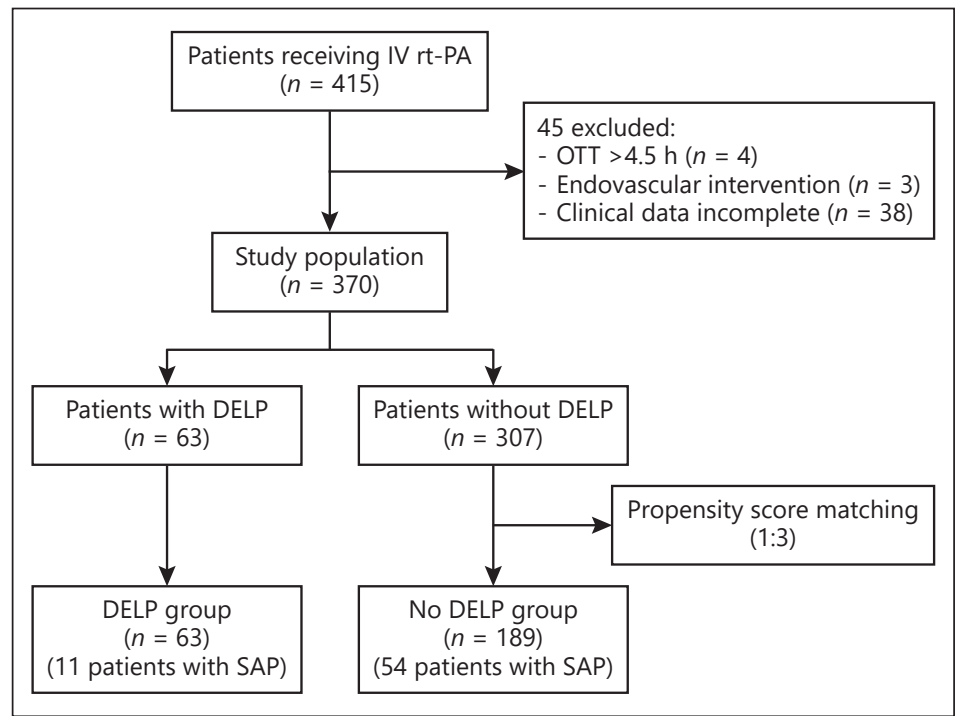

Fig. 1. Flow diagram of the participants selection. IV, intravenous; rt-PA, recombinant tissue plasminogen activator; OTT, symptom onset to thrombolysis time; DELP, delipid extracorporeal lipoprotein filter from plasma; SAP, stroke-associated pneumonia.

The rate of improvement in SAP before discharge was analyzed as an efficacy outcome in the subgroups. The clinical outcome of SAP was assessed as cured or failed according to antibacterial drug technical guiding principles for clinical trials drawn up by the CFDA Drug Approval Center [9]. Patients were considered cured if there was complete resolution of 4 aspects identified at baseline as abnormal: symptoms, signs, blood routine tests, and microbiology. Patients were considered failed if they exhibited a persistence or progression of baseline clinical signs or symptoms of infection. Patients were also considered failed if there was only a partial improvement in baseline clinical signs and symptoms of infection.

\section{Statistical Analysis}

Descriptive statistics for baseline characteristics compared patients receiving DELP treatment following IVT and patients not receiving DELP treatment. Continuous variables with normal distribution were described as means \pm SD. Continuous variables included age, NIHSS score, OTT, SBP, and DBP. Student's $t$ test was used to analyze the normally distributed continuous variables. Categorical variables were described as proportions. Categorical variables included gender, medical history, and TOAST classification. Pearson's $\chi^{2}$ tests were used to analyze categorical variables.

The outcome of serum biomarker examination and reduced NIHSS score were included in the continuous variables. In the efficacy and safety outcomes, 90-day mRS, the incidence rate of ICH, gastrointestinal bleeding, and mortality, and the SAP recovery rate were shown as categorical variables. The odds ratio (OR) and 95\% confidence interval (CI) were calculated to estimate the probabilities of a favorable 1-level shift of the mRS score with DELP treatment. $p<0.05$ was considered statistically significant. SPSS v24.0 (IBM, Amonk, NY, USA) computer software was used for the statistical analysis.

\section{Results}

Four hundred and fifteen thrombolytic patients with AIS were screened. According to the criteria, 45 patients were excluded due to different reasons: 4 with $>4.5 \mathrm{~h}$ between the onset of neurological symptoms and IVT treatment, 3 with endovascular intervention, and 38 with 


\section{Cerebrovascular Diseases Extra}

Table 1. Demographic and baseline characteristics

\begin{tabular}{|c|c|c|c|c|c|}
\hline & \multirow{2}{*}{$\begin{array}{l}\text { DELP } \\
(n=63)\end{array}$} & \multicolumn{2}{|l|}{ Before PSM } & \multicolumn{2}{|l|}{ After PSM } \\
\hline & & $\begin{array}{l}\text { NO DELP } \\
(n=307)\end{array}$ & $p$ value & $\begin{array}{l}\text { NO DELP } \\
(n=189)\end{array}$ & $p$ value \\
\hline \multicolumn{6}{|l|}{ Demographics } \\
\hline Age, years & $59.9 \pm 10.5$ & $63.3 \pm 10.8$ & $0.022^{*}$ & $61.1 \pm 11.1$ & 0.459 \\
\hline Male gender & $53(84.1)$ & $214(69.7)$ & $0.020^{*}$ & 149 (78.8) & 0.362 \\
\hline \multicolumn{6}{|l|}{ Medical history } \\
\hline Hypertension & $32(50.8)$ & 190 (61.9) & 0.102 & $103(54.5)$ & 0.610 \\
\hline Diabetes mellitus & $18(28.6)$ & $72(23.5)$ & 0.388 & $45(23.8)$ & 0.450 \\
\hline Coronary artery disease & 7 (11.1) & $61(19.9)$ & 0.102 & $30(15.9)$ & 0.355 \\
\hline Atrial fibrillation & $3(4.8)$ & $84(27.4)$ & $0.000^{*}$ & $17(9.0)$ & 0.282 \\
\hline Stroke & $4(6.3)$ & 58 (18.9) & $0.015^{*}$ & $14(7.4)$ & 0.778 \\
\hline Smoking & $9(14.3)$ & $69(22.5)$ & 0.147 & $35(18.5)$ & 0.443 \\
\hline Alcohol consumption & 7 (11.1) & $48(15.6)$ & 0.358 & $23(12.2)$ & 0.822 \\
\hline \multicolumn{6}{|l|}{ Baseline scales } \\
\hline OTT, h & $2.7 \pm 1.0$ & $3.0 \pm 0.9$ & $0.017^{*}$ & $2.9 \pm 0.9$ & 0.261 \\
\hline \multicolumn{6}{|l|}{ NIHSS score } \\
\hline At admission & $7.8 \pm 6.5$ & $11.6 \pm 8.6$ & $0.001^{*}$ & $9.0 \pm 7.5$ & 0.233 \\
\hline $24 \mathrm{~h}$ after thrombolysis & $8.3 \pm 6.5$ & $12.8 \pm 9.2$ & $0.000^{*}$ & $9.0 \pm 7.4$ & 0.471 \\
\hline $\mathrm{SBP}, \mathrm{mm} \mathrm{Hg}$ & $151.5 \pm 19.7$ & $154.9 \pm 20.3$ & 0.234 & $153.4 \pm 20.7$ & 0.531 \\
\hline DBP, mm Hg & $87.7 \pm 13.4$ & $87.5 \pm 12.3$ & 0.909 & $87.6 \pm 12.6$ & 0.955 \\
\hline \multicolumn{6}{|l|}{ TOAST classification } \\
\hline LAA & 25 (39.7) & $116(37.8)$ & 0.778 & $82(43.4)$ & 0.607 \\
\hline SAO & $22(34.9)$ & $49(16.0)$ & $0.000^{*}$ & $40(21.2)$ & 0.712 \\
\hline $\mathrm{CE}$ & $0(0.0)$ & $36(11.7)$ & $0.009 *$ & 7 (3.7) & 0.268 \\
\hline UND & $16(25.4)$ & $106(34.5)$ & 0.160 & $60(31.7)$ & 0.342 \\
\hline
\end{tabular}

Values express $n(\%)$ or mean \pm SD. DELP, delipid extracorporeal lipoprotein filter from plasma; PSM, propensity score matching; OTT, symptom onset-to-thrombolysis time; NIHSS, National Institute of Health Stroke Scale; SBP, systolic blood pressure; DBP, diastolic blood pressure; LAA, large-artery atherosclerosis; SAO, small-artery occlusion; CE, cardiogenic embolism; UND, stroke of undetermined cause. ${ }^{*} p<0.05$, compared with DELP group.

missing clinical data. Finally, 370 patients were recruited, 63 with DELP and 307 without DELP (Fig. 1). Adjusting for age, gender, OTT, SBP, DBP, NIHSS score, TOAST classification, and medical history with a ratio of $1: 3,189$ patients without DELP were matched (the NO DELP group) for the final analysis. The baseline characteristics of the 2 groups before and after PSM appear in Table 1. There was no significant difference in demographic data, medical history, baseline scales, and TOAST classification between groups after PSM.

Compared with the NO DELP group, the DELP group showed a higher proportion of 90-day mRS 0-1 (47.6 vs. 33.3\%; OR 1.818 and 95\% CI 1.018-3.246; $p=0.042$ ) and a favorable shift in 90-day mRS distribution ( $p=0.015$ ) (Table 2; Fig. 2). The NIHSS score from $24 \mathrm{~h}$ to 14 days after thrombolysis in the DELP group was significantly more reduced than that in the NO DELP group (OR 1.067; 95\% CI 1.009-1.128; $p=0.024$ ). The rate of death in the DELP group was significantly lower than in the NO DELP group $(p=0.040)$. There was no statistically significant difference in the rate of sICH or gastrointestinal bleeding.

There were 65 patients diagnosed with SAP according to the recommendations of the Pneumonia in Stroke Consensus Group [10]. There was no significant difference in demographics and baseline characteristics between the SAP subgroups, but the improvement in SAP in the DELP group was significantly greater than in the NO DELP group ( 90.9 vs. $53.7 \%$; $p=0.022$ ) (Tables 3,4 ). In patients with SAP, we found a higher proportion of favorable 
Table 2. Efficacy and safety outcomes

\begin{tabular}{lcccc}
\hline & $\begin{array}{l}\text { DELP } \\
(n=63)\end{array}$ & $\begin{array}{l}\text { NO DELP } \\
(n=189)\end{array}$ & OR (95\% CI) & $p$ value \\
\hline Efficacy outcomes & & & & \\
90-day mRS 0-1 & $30(47.6)$ & $63(33.3)$ & $1.818(1.018-3.246)$ & $0.042^{*}$ \\
90-day mRS distribution & - & - & - & $0.015^{*}$ \\
Reduced NIHSS score, mean \pm SD & $1.9 \pm 5.7$ & $-0.1 \pm 5.9$ & $1.067(1.009-1.128)$ & $0.024^{*}$ \\
\hline Safety outcomes & & & - & 0.314 \\
sICH & $0(0.0)$ & $3(1.6)$ & $0.625(0.174-2.250)$ & 0.472 \\
Gastrointestinal bleeding & $3(4.8)$ & $14(7.4)$ & - & $0.040^{*}$ \\
Mortality & $0(0.0)$ & $12(6.3)$ & - & \\
\hline
\end{tabular}

Values express $n(\%)$, unless otherwise indicated. DELP, delipid extracorporeal lipoprotein filter from plasma; OR, odds ratio; CI, confidence interval; NIHSS, National Institute of Health Stroke Scale; mRS, modified Rankin scale; sICH, symptomatic intracranial hemorrhage. ${ }^{*} p<0.05$.

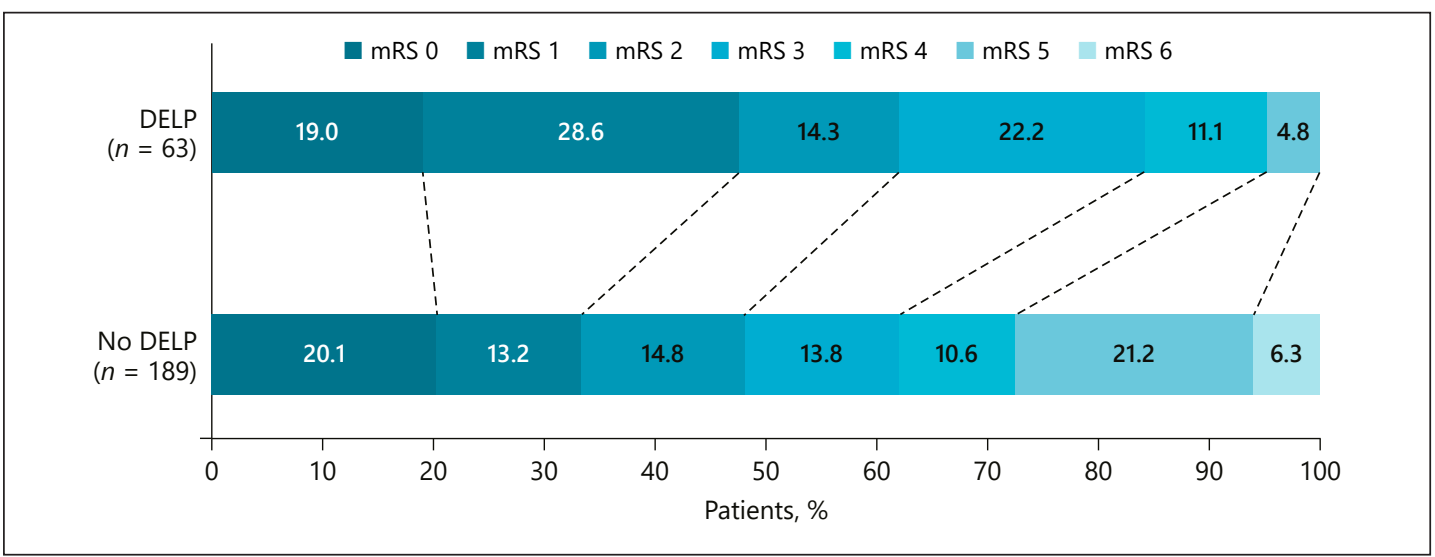

Fig. 2. Distribution of modified Rankin scale (mRS) 90 days after matching.

function at 90 days (36.4 vs. 9.3\%; $p=0.028$ ) in the DELP group. There was no statistically significant difference in the reduced NIHSS score or the rates of sICH, gastrointestinal bleeding, and mortality.

The serum biomarker results showed that DELP significantly decreased the levels of IL-1 $\beta$, E-selectin, MDA, MMP-9, TC, LDL, and FIB, and increased the level of SOD $(p<0.05)$ from baseline (Fig. 3).

\section{Discussion}

To our knowledge, our study was the first attempt to explore the efficacy and safety of DELP treatment following IVT in AIS patients. We found that there was a more favorable prognosis and decreased mortality in AIS patients who did not improve within $24 \mathrm{~h}$ after IVT in the DELP group. 
Table 3. Demographic and baseline characteristics of patients with SAP

\begin{tabular}{|c|c|c|c|}
\hline & $\begin{array}{l}\text { DELP } \\
(n=11)\end{array}$ & $\begin{array}{l}\text { NO DELP } \\
(n=54)\end{array}$ & $p$ value \\
\hline \multicolumn{4}{|l|}{ Demographics } \\
\hline Age, years & $65.5 \pm 9.2$ & $62.8 \pm 10.9$ & 0.455 \\
\hline Male gender & 9 (81.8) & $46(85.2)$ & 0.778 \\
\hline \multicolumn{4}{|l|}{ Medical history } \\
\hline Hypertension & $2(18.2)$ & $28(51.9)$ & 0.087 \\
\hline Diabetes mellitus & $4(36.4)$ & $16(29.6)$ & 0.659 \\
\hline Coronary artery disease & $1(9.1)$ & $9(16.7)$ & 0.526 \\
\hline Atrial fibrillation & $0(0.0)$ & $8(14.8)$ & 0.173 \\
\hline Stroke & $0(0.0)$ & $2(3.7)$ & 0.517 \\
\hline Smoking & $1(9.1)$ & $5(9.3)$ & 0.986 \\
\hline Alcohol consumption & $1(9.1)$ & $5(9.3)$ & 0.986 \\
\hline \multicolumn{4}{|l|}{ Baseline scales } \\
\hline OTT, h & $2.7 \pm 0.8$ & $3.0 \pm 0.8$ & 0.329 \\
\hline \multicolumn{4}{|l|}{ NIHSS score } \\
\hline At admission & $12.9 \pm 8.0$ & $13.7 \pm 8.4$ & 0.763 \\
\hline After $24 \mathrm{~h}$ & $10.9 \pm 7.0$ & $16.5 \pm 10.0$ & 0.083 \\
\hline SBP, mm Hg & $146.3 \pm 21.3$ & $152.2 \pm 19.8$ & 0.374 \\
\hline DBP, $\mathrm{mm} \mathrm{Hg}$ & $81.4 \pm 7.8$ & $88.1 \pm 12.3$ & 0.088 \\
\hline \multicolumn{4}{|l|}{ TOAST classification } \\
\hline LAA & 8 (72.7) & $28(51.9)$ & 0.349 \\
\hline SAO & $1(9.1)$ & $6(11.1)$ & 0.844 \\
\hline $\mathrm{CE}$ & $0(0.0)$ & $3(5.6)$ & 0.990 \\
\hline UND & $2(18.2)$ & $17(31.5)$ & 0.603 \\
\hline \multicolumn{4}{|l|}{ Laboratory tests } \\
\hline WBC, $\times 10^{9} / \mathrm{L}$ & $11.38 \pm 3.46$ & $11.95 \pm 3.51$ & 0.629 \\
\hline Neutrophils, $\times 10^{9} / \mathrm{L}$ & $9.49 \pm 3.15$ & $10.17 \pm 3.59$ & 0.562 \\
\hline Lymphocytes, $\times 10^{9} / \mathrm{L}$ & $1.22 \pm 0.72$ & $1.12 \pm 0.63$ & 0.647 \\
\hline Neutrophil-to-lymphocyte ratio & $9.18 \pm 4.03$ & $12.21 \pm 8.96$ & 0.280 \\
\hline $\mathrm{CRP}, \mathrm{mmol} / \mathrm{L}$ & $19.09 \pm 27.94$ & $30.34 \pm 43.05$ & 0.515 \\
\hline
\end{tabular}

Values express $n(\%)$ or mean \pm SD. SAP, stroke-associated pneumonia; DELP, delipid extracorporeal lipoprotein filter from plasma; OTT, symptom onset-to-thrombolysis time; NIHSS, National Institute of Health Stroke Scale; SBP, systolic blood pressure; DBP, diastolic blood pressure; LAA, large-artery atherosclerosis; SAO, small-artery occlusion; CE, cardiogenic embolism; UND, stroke of undetermined cause; WBC, white blood cells; CRP, C-reactive protein.

Table 4. Efficacy and safety outcomes in patients with SAP

$\begin{array}{llll}\begin{array}{l}\text { DELP } \\ (n=11)\end{array} & \begin{array}{l}\text { NO DELP } \\ (n=54)\end{array} & \text { OR }(95 \% \mathrm{CI}) & p \text { value }\end{array}$

\begin{tabular}{lcccc}
\hline Efficacy outcomes & & & & \\
Improvement in SAP & $10(90.9)$ & $29(53.7)$ & $8.621(1.031-72.114)$ & $0.022^{*}$ \\
90-day mRS 0-1 & $4(36.4)$ & $5(9.3)$ & $5.600(1.207-25.988)$ & $0.028^{*}$ \\
Reduced NIHSS score, mean \pm SD & $2.0 \pm 10.8$ & $-2.7 \pm 8.4$ & - & 0.109 \\
\hline Safety outcomes & & & & 0.423 \\
sICH & $0(0.0)$ & $3(5.6)$ & - & 0.242 \\
Gastrointestinal bleeding & $3(27.3)$ & $7(13.0)$ & $2.518(0.536-11.82)$ & 0.246 \\
Mortality & $0(0.0)$ & $6(11.1)$ & - & \\
\hline
\end{tabular}

Values express $n(\%)$, unless otherwise indicated. SAP, stroke-associated pneumonia; DELP, delipid extracorporeal lipoprotein filter from plasma; OR, odds ratio; CI, confidence interval; mRS, modified Rankin scale; NIHSS, National Institute of Health Stroke Scale; sICH, symptomatic intracranial hemorrhage. ${ }^{*} p<0.05$. 


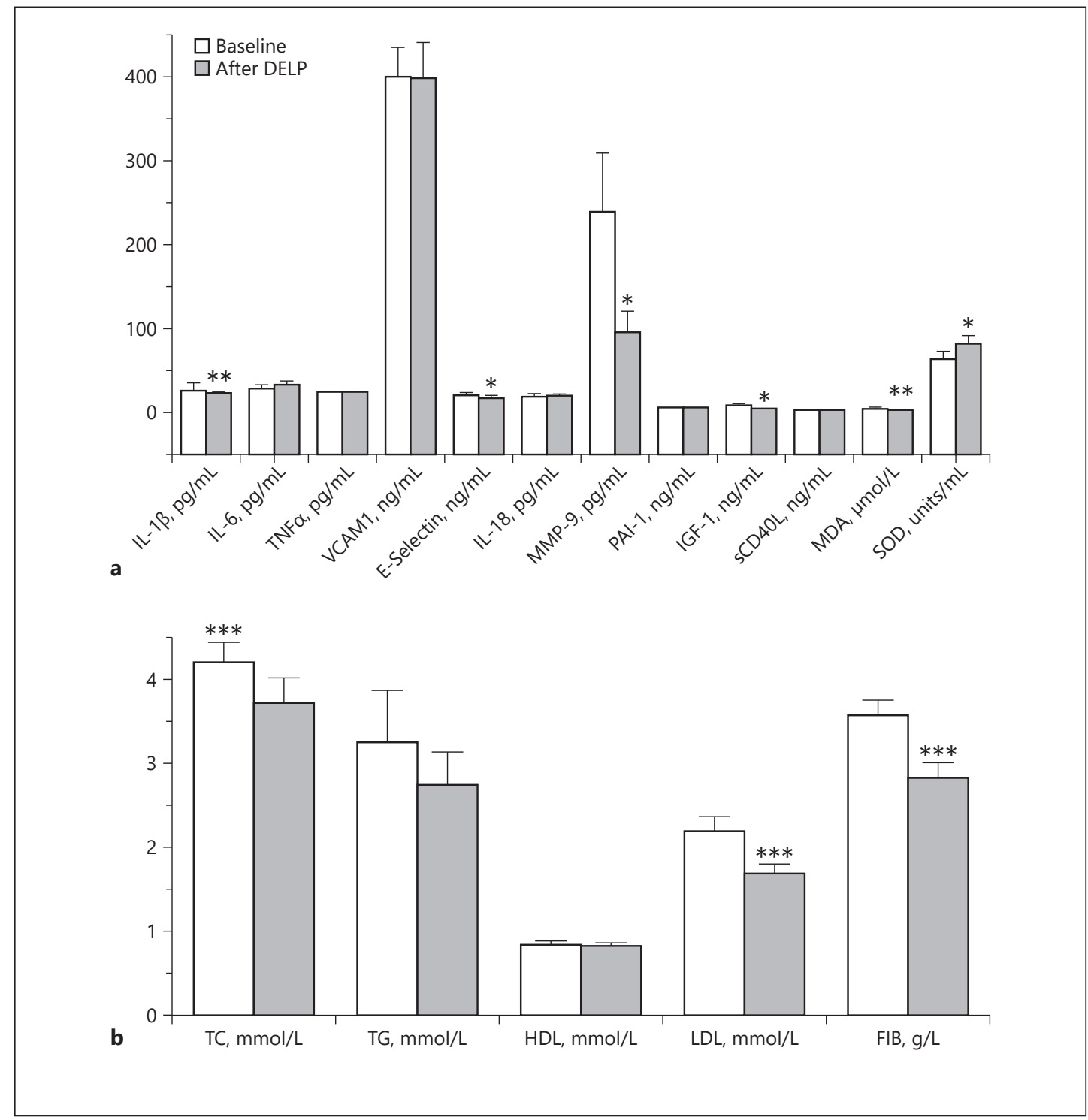

Fig. 3. a Changes in serum biomarkers prior to and after DELP treatment. b Changes in blood lipid level prior to and after DELP treatment. IL, interleukin; TNF, tumor necrosis factor; VCAM1, vascular cell adhesion molecule 1; MMP, matrix metalloprotein; PAI-1, plasminogen activator inhibitor-1; sCD40L, soluble CD40 ligand; MDA, malondialdehyde; SOD, superoxide dismutase; TC, total cholesterol; TG, triglyceride; HDL, highdensity lipoprotein; LDL, low-density lipoprotein; FIB, fibrinogen. ${ }^{*} p<0.05$; $^{* *} p<0.01$; ${ }^{* * *} p<0.001$.

Previous studies have suggested the neuroprotective effect of DELP for patients within $48 \mathrm{~h}$ after the onset of AIS. Dong et al. [8], for example, found a significant decrease in NIHSS score in AIS patients receiving DELP treatment $[11,12]$. In our study, the AIS patients who did not improve within $24 \mathrm{~h}$ after thrombolysis were chosen as the target population. Many studies have demonstrated that improvement within $24 \mathrm{~h}$ after IVT is closely associated with a good outcome [4, 5], so selecting this target population can decrease the bias and exclude the potential effect of rtPA. We found that DELP treatment produced a significant improvement of neurological function in this population and significantly decreased mortality during hospitalization. A decreased trend in SICH and gastrointestinal bleeding was also found in the DELP 
group. Taken together, our results suggest a possible neuroprotective effect of DELP in this population.

Similar to HELP, DELP may exert multiple mechanisms to treat AIS [6, 7], but, to date, the neuroprotective mechanism of DELP has not yet been fully determined. We found that the levels of IL-1 $\beta$, E-selectin, and MDA decreased significantly, and that of SOD increased significantly after DELP treatment, which points to anti-inflammatory and free radical-scavenging effects of DELP. Additionally, we found the levels of TC, LDL, and FIB significantly decreased after DELP treatment. Higher FIB results in higher blood viscosity, which potentially compromises the cerebral vascular blood flow [13]. Decreasing FIB levels appear to be associated with a favorable functional outcome of AIS [14]. We inferred that the neuroprotective effect of DELP treatment may be partially mediated by improving hemorheology [6]. It was unexpected that the level of MMP-9 was significantly decreased after DELP (from 250 to $100 \mathrm{ng}$ / $\mathrm{mL}$ ). Previous studies found that MMP-9 increased after IVT treatment, and an increased MMP-9 may impair the functional integrity of the whole neurovascular unit, which can result in greater brain edema, cerebral hemorrhagic transformation (HT), and poor neurological outcomes in stroke patients receiving IVT treatment [15-17]. We argued that improved prognosis and short-term neurological function, and decreased mortality could be attributable to multiple mechanisms, e.g., improved hemorheology and the anti-inflammatory and free radical-scavenging effects of DELP [18]. MMP-9 is closely related to HT [19-22]. We performed DELP >1 day after thrombolysis; HT usually occurs within 24-36 h afterwards. It is a good idea to perform DELP during or immediately after thrombolysis to determine the effect on thrombolysis-related HT.

Considering that SAP was the most frequent complication after stroke and the antiinflammatory effect of DELP treatment, we further analyzed the efficacy of DELP treatment in the patients with SAP [23]. It was interesting that the rate of recovery from SAP in the DELP group was significantly higher than in NO DELP group. We argue that this improvement can be attributed to the anti-inflammatory effects of DELP, e.g., a decreased level of IL-1 $\beta$. We also found a higher proportion of favorable functional outcome in patients with SAP in the DELP group, in line with a previous study showing that SAP is linked to a poor outcome (mRS 3-6) at 3 months [24].

We acknowledge that our study has several limitations. First, although the data were matched by propensity score, there may have been a confounding bias due to the retrospective nature of the study. Second, it was a small-sample, single-center study, which limited the analysis of the relationship between the effect and the beginning of DELP. Given that neuroinflammation occurred immediately after stroke onset, we argued that starting DELP earlier may be better, e.g., performed immediately after IVT or endovascular treatment. Last, the study did not include AIS patients with neurological function improvements within $24 \mathrm{~h}$ after receiving IVT treatment, so the effect of DELP treatment following IVT treatment in these patients was not known. Prospective, randomized controlled trials with larger samples should be done to verify the exact effect of DELP treatment for AIS patients receiving IVT.

\section{Conclusion}

We showed that DELP treatment can be safe and feasible. It was associated with improved favorable prognosis and decreased mortality in the stroke patients with no improvement within $24 \mathrm{~h}$ after intravenous thrombolysis, possibly through multiple mechanisms. Randomized controlled trials should be urgently conducted to determine the efficacy of DELP treatment in this population.

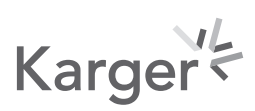




\section{Cerebrovascular Diseases Extra}

\begin{tabular}{l|l}
\hline Cerebrovasc Dis Extra 2020;10:148-158 \\
\hline DOI: 10.1159/000511050 & $\begin{array}{l}\text { ○ 2020 The Author(s). Published by S. Karger AG, Basel } \\
\text { www.karger.com/cee }\end{array}$ \\
\hline
\end{tabular}

Cui et al.: DELP Treatment after Intravenous Thrombolysis for AIS

\section{Statement of Ethics}

The study was exempt from approval by the Ethics Committee of General Hospital of Northern Theater Command. Due to the retrospective nature, the study waived the need for patient informed consent.

\section{Conflict of Interest Statement}

The authors declare that they have no competing interests.

\section{Funding Sources}

This study was supported by grants from the Science and Technology Project Plan of Liao Ning Province (2018225023 and 2019JH2/10300027).

\section{Author Contributions}

Y.C. and Z.H.Z. collected the clinical data of patients, X.W.H. performed the data analysis, Y.C. drafted the manuscript; H.S.C. contributed to the study design and critically edited the manuscript.

\section{References}

1 Hacke W, Kaste M, Bluhmki E, Brozman M, Dávalos A, Guidetti D, et al.; ECASS Investigators. Thrombolysis with alteplase 3 to 4.5 hours after acute ischemic stroke. N Engl J Med. 2008 Sep;359(13):1317-29.

2 National Institute of Neurological Disorders and Stroke rt-PA Stroke Study Group. Tissue plasminogen activator for acute ischemic stroke. N Engl J Med. 1995 Dec;333(24):1581-7.

3 Wahlgren N, Ahmed N, Dávalos A, Ford GA, Grond M, Hacke W, et al.; SITS-MOST investigators. Thrombolysis with alteplase for acute ischaemic stroke in the Safe Implementation of Thrombolysis in Stroke-Monitoring Study (SITS-MOST): an observational study. Lancet. 2007 Jan;369(9558):275-82.

4 Brown DL, Johnston KC, Wagner DP, Haley EC Jr. Predicting major neurological improvement with intravenous recombinant tissue plasminogen activator treatment of stroke. Stroke. 2004 Jan;35(1):147-50.

5 Saposnik G, Di Legge S, Webster F, Hachinski V. Predictors of major neurologic improvement after thrombolysis in acute stroke. Neurology. 2005 Oct;65(8):1169-74.

6 Walzl M, Schied G, Walzl B. Effects of ameliorated haemorheology on clinical symptoms in cerebrovascular disease. Atherosclerosis. 1998 Aug;139(2):385-9.

7 Jaeger BR. The HELP system for the treatment of atherothrombotic disorders: a review. Ther Apher Dial. 2003 Aug; 7(4):391-6.

8 Dong Q, Fang K, Wang X, et al. Initial Analysis of Multicenter Randomized Controlled Clinical Trial of DELP System in Treating Patients with Acute Cerebral Infarction. Neural Injury Funct Reconstr J. 2008 Jan;3(1):8-11.

9 Sun T, Sun L, Wang R, Ren X, Sui DJ, Pu C, et al. Clinical efficacy and safety of moxifloxacin versus levofloxacin plus metronidazole for community-acquired pneumonia with aspiration factors. Chin Med J (Engl). 2014; 127(7):1201-5.

10 Smith CJ, Kishore AK, Vail A, Chamorro A, Garau J, Hopkins SJ, et al. Diagnosis of Stroke-Associated Pneumonia: Recommendations from the Pneumonia in Stroke Consensus Group. Stroke. 2015 Aug;46(8):2335-40.

11 Liu MY, Guan YT, Hou XJ, et al. Obseration of Curative Effect of Extracorporeal Lipoprotein Filter from Plasma Delipid plus Therapy on Acute Cerebral Infarction. J Clin Neurol. 2008 Dec;21(6):460-2.

12 Fang K, Chen SF, Xiang H, et al. Subgroup Analysis of the Efficacy of Delipid Extracossoseal Lipoprotein Filter from Plasma for Acute Cerebral Ischemic Stroke. Chin J Cerebrovasc Dis. 2009 Feb;3(1):26-32.

13 del Zoppo GJ, Levy DE, Wasiewski WW, Pancioli AM, Demchuk AM, Trammel J, et al. Hyperfibrinogenemia and functional outcome from acute ischemic stroke. Stroke. 2009 May;40(5):1687-91.

14 Sherman DG, Atkinson RP, Chippendale T, Levin KA, Ng K, Futrell N, et al.; for the STAT Participants. Intravenous ancrod for treatment of acute ischemic stroke: the STAT study: a randomized controlled trial. Stroke Treatment with Ancrod Trial. JAMA. 2000 May;283(18):2395-403. 


\section{Cerebrovascular Diseases Extra}

\begin{tabular}{l|l}
\hline Cerebrovasc Dis Extra 2020;10:148-158 \\
\hline DOI: 10.1159/000511050 & $\begin{array}{l}\text { @ 2020 The Author(s). Published by S. Karger AG, Basel } \\
\text { www.karger.com/cee }\end{array}$ \\
\hline
\end{tabular}

Cui et al.: DELP Treatment after Intravenous Thrombolysis for AIS

15 Rosenberg GA, Estrada EY, Dencoff JE. Matrix metalloproteinases and TIMPs are associated with blood-brain barrier opening after reperfusion in rat brain. Stroke. 1998 Oct;29(10):2189-95.

16 Castellanos M, Leira R, Serena J, Pumar JM, Lizasoain I, Castillo J, et al. Plasma metalloproteinase-9 concentration predicts hemorrhagic transformation in acute ischemic stroke. Stroke. 2003 Jan;34(1):40-6.

17 Horstmann S, Kalb P, Koziol J, Gardner H, Wagner S. Profiles of matrix metalloproteinases, their inhibitors, and laminin in stroke patients: influence of different therapies. Stroke. 2003 Sep;34(9):2165-70.

18 Charles C. Esenwa, Mitchell S. Elkind. Inflammatory Risk Factors, Biomarkers and Associated Therapy in Ischaemic Stroke. Nature Neurology. 2016 Oct;12(10):594-604.

19 Castellanos M, Sobrino T, Millán M, García M, Arenillas J, Nombela F, et al. Serum cellular fibronectin and matrix metalloproteinase- 9 as screening biomarkers for the prediction of parenchymal hematoma after thrombolytic therapy in acute ischemic stroke: a multicenter confirmatory study. Stroke. 2007 Jun;38(6):1855-9.

20 Lapchak PA, Chapman DF, Zivin JA. Pharmacological effects of the spin trap agents N-t-butyl-phenylnitrone (PBN) and 2,2,6, 6-tetramethylpiperidine-N-oxyl (TEMPO) in a rabbit thromboembolic stroke model: combination studies with the thrombolytic tissue plasminogen activator. Stroke. 2001 Jan;32(1):147-53.

21 Zhang L, Zhang ZG, Zhang RL, Lu M, Adams J, Elliott PJ, et al. Postischemic (6-Hour) treatment with recombinant human tissue plasminogen activator and proteasome inhibitor PS-519 reduces infarction in a rat model of embolic focal cerebral ischemia. Stroke. 2001 Dec;32(12):2926-31.

22 Castellanos M, Leira R, Serena J, Blanco M, Pedraza S, Castillo J, et al. Plasma cellular-fibronectin concentration predicts hemorrhagic transformation after thrombolytic therapy in acute ischemic stroke. Stroke. 2004 Jul; 35(7):1671-6.

23 Katzan IL, Cebul RD, Husak SH, Dawson NV, Baker DW. The effect of pneumonia on mortality among patients hospitalized for acute stroke. Neurology. 2003 Feb;60(4):620-5.

24 Mengel A, Ulm L, Hotter B, Harms H, Piper SK, Grittner U, et al. Biomarkers of immune capacity, infection and inflammation are associated with poor outcome and mortality after stroke - the PREDICT study. BMC Neurol. 2019 Jul;19(1):148. 